\title{
Semi-topological properties
}

\section{by}

\section{S. Gene Crossley and S. K. Hildebrand (Lubbock, Tex.)}

0. Introduction. In [1], Norman Levine defined a semi-open set in a topological space as a set $A$ such that there exists an open set $O$ so that $O \subseteq A \subseteq \bar{O}$, where ( $\overline{()}$ denotes closure in the topological space. He also defined a function to be semi-continuous if and only if the inverses of open sets are semi-open. Also in [1], among others, the following two results were established:

THEOREM 0.1. Let $(X, \tau)$ be a topological space. Then:

(1) $\tau \subseteq \mathrm{SO}(X, \tau)$ where $\mathrm{SO}(X, \tau)$ denotes the class of semi-open sets in $(X, \tau)$, and

(2) for $A \in \mathrm{SO}(X, \tau)$ and $A \subseteq B \subseteq \bar{A}$, then $B \in \mathrm{SO}(X, \tau)$.

THEOREM 0.2. Let $f: X \rightarrow Y$ be continuous and open where $X$ and $Y$ are topological spaces. Let $A \in \mathrm{SO}(X)$. Then $f(A) \in \mathrm{SO}(\bar{X})$.

In [2], the authors defined a set to be semi-closed if and only if its complement is semi-open. Semi-closure and semi-interior were defined in a manner analogous to closure and interior. Also in [2], among others, the following four results were established:

THEOREM 0.3. In a topological space all nonvoid semi-open sets must contain nonvoid open sets.

THEOREM 0.4. (1) $A$ is semi-open if and only if $A_{0}=A$, where ( $)_{0}$ denotes the semi-interior in the topological space.

(2) $A$ is semi-closed if and only if $\underline{A}=A$, where ( ) denotes semiclosure in the topological space.

THEOREM 0.5. If $A$ is open and $S$ is semi-open, then $(A \cap S)$ is semi-open.

THEOREM $0.6 .(\underline{X-(\bar{A}-A)})=X$.

Then in [2], an abstract operator approach was introduced. A presemi-closure operator, usually denoted by ( $)_{c}$, was defined, and it was demonstrated that if a pre-semi-closure operator is related to a Kuratowski closure operator in a particular fashion, then the pre-semi-closure 
operator corresponds exactly to the semi-closure in the topological space generated by the Kuratowski closure operator. A method by which a Kuratowski closure operator may be constructed from a pre-semi-closure operator was developed: First it was shown that for every set $A$ there exists a minimal set $D_{A}$ such that $\left(A \cup D_{A} \cup B\right)_{c}=\left(A \cup D_{A} \cup B_{c}\right)$ for every subset $B$ of the comprehending set. Then, defining $A^{k}=\left(A \cup D_{A}\right)$ it was shown that ()$^{k}$ is a Kuratowski closure operator. A criterion was found to guarantee that the pre-semi-closure operator is properly related to the Kuratowski closure operator constructed from it, in which case the pre-semi-closure operator is called a semi-closure operator. And, finally, it was shown that the topology generated by the constructed Kuratowski closure operator is the finest topology for which a semiclosure operator is the semi-closure in the topological space.

Finally, in [3], it was shown that:

THEOREM 0.7. $(\underline{A})_{0} \supseteq(\bar{A})^{0}$.

1. Irresolute functions. In this section, various types of functions are studied. The first kind of function to be considered is one for which inverses of semi-open sets are semi-open.

Definition 1.1. A function $f: X \rightarrow Y$ is said to be irresolute if and only if, for any semi-open subset $S$ of $Y, f^{-1}(S)$ is semi-open in $X$.

The following theorem is a result from elementary topology, and is stated without proof.

THEOREM 1.1. If $f: X \rightarrow Y$ is continuous and open, then $f^{-1}(\bar{A})=\overline{f^{-1}(A)}$.

THEOREM 1.2. Let $f: X \rightarrow Y$ be continuous and open where $X$ and $Y$ are topological spaces, then $f$ is irresolute.

Proof. If $A \in \mathrm{SO}(Y)$, then there is an open set $O \subset Y$ such that $O \subseteq A \subseteq \bar{O}$. By Theorem 1.1, $f^{-1}(\bar{O})=\overline{f^{-1}(O)}$. Also, $f^{-1}(O) \subseteq f^{-1}(A) \subseteq f^{-1}(\bar{O})$ $=\bar{f}^{-1}(O)$, and since $f$ is continuous, $f^{-1}(O)$ is open. Thus $f^{-1}(A)$ is semi-open.

EXAMPLE 1.1. A continuous, irresolute function need not be open. Let $X=\{a, b, c\}$, and consider the topologies

$$
\tau^{*}=\{\varnothing,\{a\},\{a, b\}, X\} \quad \text { and } \quad \tau=\{\varnothing,\{a\},\{a, b\},\{a, c\}, X\} .
$$

It was demonstrated in [2] that $\mathrm{SO}(X, \tau)=\operatorname{SO}\left(X, \tau^{*}\right)$. Let $f:(X, \tau)$ $\rightarrow\left(X, t^{*}\right)$ be defined by $f(x)=x$ for all $x \in X$. Then clearly $f$ is continuous and irresolute, bat not open.

Later on there will be abundant examples of this fact, but this is one of the simplest.

THEonem 1.3. Let $C(X, Y), S C(X, Y)$, and $I(X, Y)$ denote, respectively, the classes of continuous, semi-continuous, and irresolute functions from $X$ to $Y$, where $X$ and $Y$ are topological spaces. Then $C(X, Y)$ $\subseteq S C(X, Y)$, and $I(X, Y) \subseteq S C(X, Y)$.

Proof. $C(X, Y) \subseteq S O(X, X)$ because if the inverses of open sets are open, it follows by Theorem 0.1 that the inverses of open sets are semi-open.

$I(X, Y) \subseteq S C(X, Y)$ because if the inverses of semi-open sets are semi-open, it follows by Theorem 0.1 that the inverses of open sets are semi-open.

There will be numerous cases later in which it will be seen that irresolute functions are not necessarily continuous. Neither are continuous functions necessarily irresolute.

THeorem 1.4. A function $f: S \rightarrow T$ is irresolute if and only if, for every semi-closed subset $H$ of $T, f^{-1}(H)$ is semi-closed in $S$.

Proof. Necessity. If $f: S \rightarrow T$ is irresolute, then for every semi-open subset $Q$ of $T, f^{-1}(Q)$ is semi-open in $S$. If $H$ is any semi-closed subset of $T$, then $(T-H)$ is semi-open. Thus $f^{-1}(T-H)$ is semi-open, but $f^{-1}(T-H)=\left(S-f^{-1}(H)\right)$ so that $f^{-1}(H)$ is semi-closed.

Sufficiency. If, for all semi-closed subsets $H$ of $T, f^{-1}(H)$ is semiclosed in $S$, and if $Q$ is any semi-open subset of $T$, then $(T-Q)$ is semiclosed. Also, $f^{-1}(T-Q)=\left(S-f^{-1}(Q)\right)$ is semi-closed. Thus $f^{-1}(Q)$ is semi-open.

THEOREM 1.5. A function $f: S \rightarrow T$, where $S$ and $T$ are topological spaces, is irresolute if and only if, for every subset $A$ of $S, f(\underline{A}) \subseteq \underline{f(A)}$.

Proof. Necessity. If $A \subseteq S$, then consider $f(A)$ which is semiclosed in T. Thus by Theorem 1.4, $f^{-1}(\underline{f(A)})$ is semi-closed in S. Furthermore, $A \subseteq f^{-1}(f(A)) \subseteq f^{-1}(\underline{f(A))})$. Therefore, by the definition of semiclosure, $\underline{A} \subseteq f^{-1}(\underline{f(A))}$, and consequently,

$$
f(\underline{A}) \subseteq f\left(f^{-1}(\underline{f(A)})\right)=(\underline{f(A)} \cap f(S)) \subseteq \underline{f(A)} .
$$

Sufficiency. If $H$ is semi-closed in $T$, consider $f^{-1}(H)$. Note that

$$
f\left(\underline{f^{-1}(H)}\right) \subseteq \underline{f\left(f^{-1}(H)\right)}=\underline{(H \cap f(S))} \subseteq \underline{\underline{H}}=H .
$$

Hence $f^{-1}(H) \subseteq f^{-1}(\dot{H})$, so that $f^{-1}(H)=f^{-1}(H)$; and by Theorem 0.4, $f^{-1}(H)$ is semi-closed. Thus $f$ is irresolute by Theorem 1.4.

THEOREM 1.6. A function $f: X \rightarrow Y$ is irresolute if and only if, for all $B \subseteq \bar{\subseteq}, \underline{f^{-1}(B)} \subseteq f^{-1}(\underline{B})$.

Proof. Necessity. $\underline{B}$ is semi-closed in $Y$, so that $f^{-1}(\underline{B})$ is semiclosed in $X$. Since $f^{-1}(B) \subseteq f^{-1}(\underline{B})$, it follows by the definition of semi-

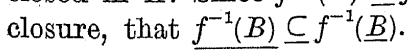


Sufficiency. If $B$ is semi-closed in $Y$, then $B=\underline{B}$. By hypothesis,

$$
f^{-1}(B) \subseteq \underline{f^{-1}(B)} \subseteq f^{-1}(\underline{B})=f^{-1}(B) ;
$$

that is, $f^{-1}(B)=f^{-1}(B)$. Thus by Theorem $0.4, f^{-1}(B)$ is semi-closed, and by Theorem 1.4, $f$ is irresolute.

THEOREM 1.7. If $f: X \rightarrow Y$ and $g: Y \rightarrow Z$ are both irresolute, then $g(f): X \rightarrow Z$ is irresolute.

Proof. If $A \subseteq Z$ is semi-open, then $g^{-1}(A)$ is semi-open and $f^{-1}\left(g^{-1}(A)\right)$ is semi-open since $g$ and $f$ are irresolute. Thus $(g(f))^{-1}(A)=f^{-1}\left(g^{-1}(A)\right)$ is semi-open, and $g(f)$ is irresolute.

DeFINITION 1.2. Let $X$ and $Y$ be topological spaces, a function $f: X \rightarrow Y$ is said to be pre-semi-open if and only if, for all $A \in \mathrm{SO}(X)$, $f(A) \in \mathbb{S O}(Y)$.

THEOREM 1.8. If $f: X \rightarrow Y$ is continuous and open, then $f$ is irresolute and pre-semi-open.

Proof. This result follows from Theorem 0.2 and Theorem 1.2.

Defrinition 1.3. Let $X$ and $Y$ be topological spaces. $X$ and $Y$ are said to be semi-homeomorphic if and only if there exists a function $f: X \rightarrow Y$ such that $f$ is one-to-one, onto, irresolute, and pre-semi-open. Such an $f$ is called a semi-homeomorphism.

THeorem 1.9. If $f: X \rightarrow Y$ is a homeomorphism, then $f$ is a semihomeomorphism.

Proof. The proof follows directly from Theorem 1.8.

EXAMPLE 1.2. A semi-homeomorphism need not be a homeomorphism. Consider $f:(X, \tau) \rightarrow\left(X, \tau^{*}\right)$ as in Example 1.1, then $f$ is a semi-homeomorphism, but $f$ is not a homeomorphism.

Remark 1.1. The image of a $T_{0}$ space under a semi-homeomorphism is not necessarily $T_{0}$, as is illustrated in the following example.

EXAMPLE 1.3. Let $X=[0,1)$, and let $\tau_{1}=(\{\varnothing, X\} \cup\{[0, a): 0<a$ $\leqslant 1\})$, then $\tau_{1}$ is a topology and a nonvoid set $A$ is in $\operatorname{SO}\left(X, \tau_{1}\right)$ if and only if $[0, b) \subseteq A$ for some $b, 0<b \leqslant 1$. Furthermore, $\left(X, \tau_{1}\right)$ is a $T_{0}$ space. Let $\tau_{2}$ consist of $\varnothing, X$, and all sets of the form $\left[0,2^{-n}\right)$ for $n=1,2,3, \ldots$, then $\tau_{2}$ is a topology, and SO $\left(X, \tau_{2}\right)=\operatorname{SO}\left(X, \tau_{1}\right)$. Define $f:\left(X, \tau_{1}\right) \rightarrow\left(X, \tau_{2}\right)$ by $f(x)=x$ for all $x \in X$. Then $f$ is a semi-homeomorphism, but $\left(X, \tau_{2}\right)$ is not a $T_{0}$ space.

Remark 1.2. The image of a $T_{1}$ space under a semi-homeomorphism is not necessarily a $T_{1}$ space; as is illustrated in the following example.

EXAMPLe 1.4. Let $X=(R \times R)$ where $R$ denotes the set of real numbers, and let $\tau_{1}$ be defined to consist of $\emptyset$ together with all subsets of $X$ whose complements are subsets of a finite number of lines parallel to the $x$-axis. Note that $\operatorname{SO}\left(X, \tau_{1}\right)=\tau_{1}$. Let $\tau_{2}$ consist of $\emptyset$ together with all subsets of $X$ whose complements are a finite number of lines parallel to the $x$-axis. Note that $\mathrm{SO}\left(X, \tau_{2}\right)=\mathrm{SO}\left(X, \tau_{1}\right)$. Furthermore, defining $f:\left(X, \tau_{1}\right) \rightarrow\left(X, \tau_{2}\right)$ by $f(p)=p$ for $p \in X$, we see that $f$ is a semihomeomorphism. Observe that $\left(X, \tau_{1}\right)$ is a $T_{1}$ space whereas $\left(X, \tau_{2}\right)$ is not.

It will be shown in the next section that the image of a $T_{2}$ space under a semi-homeomorphism is $T_{2}$.

THeOREM 1.10. If $f: X \rightarrow Y$ is a semi-homeomorphism, then $\underline{f^{-1}(B)}=f^{-1}(\underline{B})$ for all $B \subseteq Y$.

Proof. By Theorem 1.6, $f^{-1}(B) \subseteq f^{-1}(\underline{B})$ since $f$ is irresolute. $f^{-1}$ is an irresolute function, so by Theorem 1.5, $f^{-1}(\underline{B}) \subseteq \underline{\subseteq f^{-1}(B)}$. Thus, equality holds.

CoROLLARY 1.1. If $f: X \rightarrow Y$ is a semi-homeomorphism, then $f(B)=f(\underline{B})$ for all $B \subset X$.

CoROLLARX 1.2. If $f: X \rightarrow Y$ is a semi-homeomorphism, then $f\left(B_{0}\right)$ $=(f(B))_{0}$ for all $B \subseteq X$.

Proof. $B_{0}=(X-\underline{(X-B)})$. Thus,

$f\left(B_{0}\right)=(Y-f \underline{(X-B)})=(Y-\underline{f(X-B)})=(Y-(\underline{Y-f(B)}))=(f(B))_{0}$.

COROLLART 1.3. If $f: X \rightarrow Y$ is a semi-homeomorphism, then $f^{-1}\left(B_{0}\right)$ $=\left(f^{-1}(B)\right)_{0}$ for all $B \subseteq \bar{Y}$.

THeOREM 1.11. $(\underline{A})_{0}=\emptyset$ if and only if $A$ is nowhere dense.

Proof. Necessity. By Theorem $0.7,(\bar{A})^{0} \subseteq(\underline{A})_{0}$. Thus, if $(\underline{A})_{0}=\emptyset$, it follows immediately that $(\bar{A})^{0}=\varnothing$; that is, $\bar{A}$ is nowhere dense.

Sufficiency. If $A$ is nowhere dense, then $(\bar{A})^{0}=\varnothing$. But $\underline{A} \subseteq \bar{A}$, so that $\varnothing$ is the only open set contained in $\underline{A}$. Thus by Theorem $0.3, \varnothing$ is the only semi-open set contained in $\underline{A}$. Therefore, $(\underline{A})_{0}=\emptyset$.

THeorem 1.12. If $f: X \rightarrow \bar{X}$ is a semi-homeomorphism and $A \subseteq X$ is nowhere dense in $X$, then $f(A)$ is nowhere dense in $Y$.

Proof. By Theorem 1.11, since $A$ is nowhere dense, $(\underline{A})_{0}=\emptyset$. Now consider $f(A)$. Note that by Corollary 1.1, $\underline{f(A)}=f(\underline{A})$. Thus $(f(A))_{0}$ $=(f(\underline{A}))_{0}=f\left((\underline{A})_{0}\right)$ by Corollary 1.2. Hence $(\underline{f(A)})_{0}=f(\varnothing)=\varnothing$, and $f(A)$ is nowhere dense by Theorem 1.11 .

THEOREM 1.13. Semi-homeomorphic is an equivalence relation between topological spaces.

Proof. Reflexivity and symmetry are immediate and transitivity follows from Theorem 1.7. 
DEFInITION 1.4. A property which is preserved under semi-homeomorphisms is said to be a semi-topological property.

Examples 1.3 and 1.4 show that $T_{0}$ and $T_{1}$ are not semi-topological properties. However in the next section it will be shown that $T_{2}$ is a semitopological property.

Remark 1.3. Regularity, complete normality, normality, $T_{3}, T_{4}, T_{5}$, paracompactness, Lindelöf, and metrizability are not semi-topological properties, as is shown in the following example.

ExaMrLe 1.5. Let $X$ be the closed upper half-plane of $R^{2}$, and let $Y$ be the open upper half-plane. Let $\tau$ be the usual relative topology on $X$.

Let $\tau^{*}$ be generated by the usual open spheres about points in $Y$, and for basic open neighborhoods of points $p$ in $(X-Y)$, take $\left\{\left(\left(S_{\varepsilon}(p) \cap Y\right) \cup\{p\}\right)\right\}$ for all $\varepsilon>0$.

Note that $\tau^{*}$ is finer than $\tau$, and $\tau$ is $T_{2}$, regular, completely normal, normal, $T_{3}, T_{4}, T_{5}$, paracompact, Lindelöf, and metrizable. However, $\tau^{*}$ is $T_{2}$, but not regular, completely normal, normal, $T_{3}, T_{4}, T_{5}$, paracompact, Lindelöf, nor metrizable. Furthermore, it will be shown that $\mathrm{SO}(X, \tau)=\mathrm{SO}\left(X, \tau^{*}\right)$ so that the identity is a semi-homeomorphism.

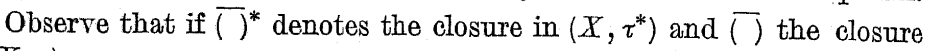
in $(X, \tau)$,

$$
\bar{B}^{*}=\left\{\begin{array}{lll}
\bar{B} & \text { if } & B \subseteq Y, \\
B & \text { if } & B \subseteq(X-Y) .
\end{array}\right.
$$

However, there are no open subsets of $(X-Y)$ in either $\tau^{*}$ or $\tau$. For any open set $U$ in $\tau^{*}, U=\left(U_{1} \cup C\right)$ where $U_{1} \subseteq Y$ and $C \subseteq(X-Y)$. Furthermore, $U_{1} \in \tau$ because for points in $Y$, the bases of $\tau$ and $\tau^{*}$ coincide. In addition, $C \subseteq \overline{U_{1}}$ because if $p \in C$, then $p \in U$ which is open in $\tau^{*}$. Thus there is an $\bar{\varepsilon}>0$ such that $\left[\left(\left(S_{\varepsilon}(p)\right) \cap Y\right) \cup\{p\}\right] \subseteq U$. Therefore $\left(S_{\varepsilon}(p) \cap\right.$ $\cap Y \cap U) \neq \varnothing$, but $(Y \cap U)=U_{1}$. Clearly it follows that for any $\varepsilon>0, S_{\varepsilon}(p)$ intersects $U_{1}$ in a nonvoid set, thus $p \in \overline{U_{1}}$. Hence $U_{1} \subseteq Y$, $U_{1} \in \tau$, and $C \subseteq\left((X-Y) \cap \overline{J_{1}}\right)$. Note that $C$ might very well be empty. Thus,

$\bar{U}^{*}=\overline{\left(\overline{U_{1} \cup C}\right)^{*}}=\left(\bar{U}_{1}^{*} \cup \bar{C}^{*}\right)=\left(\overline{U_{1} \cup C}\right)=\overline{\bar{U}_{1}}=\left(\overline{U_{1}} \cup \bar{C}\right)=\left(\overline{U_{1} \cup C}\right)=\bar{U}$, for all $\bar{U} \in \tau^{*}$.

If $A \in \mathrm{SO}(X, \tau)$, then there exists $O \in \tau$ such that $O \subseteq A \subseteq \bar{O}=\bar{O}^{*}$ since $\tau \subseteq \tau^{*}$. Thus $A \in \operatorname{SO}\left(X, \tau^{*}\right)$, and $\operatorname{SO}(X, \tau) \subseteq \operatorname{SO}\left(X, \tau^{*}\right)$.

If $\bar{A} \in \mathrm{SO}\left(X, \tau^{*}\right)$, then there exists $U \in \tau^{*}$ such that $U \subseteq A \subseteq \bar{U}^{*}$. As before, $U=\left(U_{1} \cup C\right)$ where $U_{1} \in \tau$ and $\bar{U}^{*}=\overline{U_{1}}$, thus $U_{1} \subseteq \underline{A} \subseteq \overline{\bar{U}_{1}}$ so that $A \in \mathrm{SO}(X, \tau)$.

Hence $\mathrm{SO}(X, \tau)=\mathrm{SO}\left(X, \tau^{*}\right)$.
Remark 1.4. Example 1.5 also illustrates that the images of compact sets are not necessarily compact, and the images of connected sets are not necessarily connected under a semi-homeomorphism. Note that $(X-Y)$ is connected in $(X, \tau)$ but not connected in $\left(X, \tau^{*}\right)$. Furthermore, if $p \in(X-Y)$, the set consisting of the usual closed sphere of radius $r$ about $p$, intersected with $(X-Y)$, is compact in $(X, \tau)$ but is not compact in $\left(X, \tau^{*}\right)$.

THEOREM 1.14. The property that a topological space be of the first category is a semi-topological property.

Proof. If $X$ is of the first category, then $X=\bigcup_{j=1}^{\infty} A_{j}$, where each $A_{j}$ is nowhere dense. Thus, if $f: X \rightarrow Y$ is a semi-homeomorphism,

$$
Y=f(X)=f\left(\bigcup_{j=1}^{\infty} A_{j}\right)=\bigcup_{j=1}^{\infty} f\left(A_{j}\right),
$$

and each $f\left(A_{j}\right)$ is nowhere dense by Theorem 1.12. Thus $I$ is of the first category.

COROLLARY 1.4. The property that a topological space be of the second category is a semi-topological property.

Remark 1.5. Example 1.5 also shows that second countability is not a semi-topological property.

THEOREM 1.15. A semi-topological property is a topological property.

Proof. This result follows since by Theorem 1.9, every homeomorphism is a semi-homeomorphism.

Remark 1.6. Compactness is not a semi-topological property as was illustrated in Example 1.3. In Example 1.3, if $f:\left(X, \tau_{1}\right) \rightarrow\left(X, \tau_{2}\right)$ is defined to be the identity, then $f$ is a semi-homeomorphism while $\left(X, \tau_{1}\right)$ is not compact and $\left(X, \tau_{2}\right)$ is compact.

It will be shown in the next section that connectedness is a semitopological property. Thus, by remark 1.4, if $f: X \rightarrow Y$ is a semi-homeomorphism and $A \subseteq X$, then $f \mid A: A \rightarrow f(A)$ is not necessarily a semihomeomorphism.

At this time it is desirable to construct the topology generated by the usual semi-closure on the set of real numbers by the method developed in [2]. In the next two theorems let $R$ denote the set of real numbers, and let. $(\overline{)}$ and ( ) denote the usual closure and semi-closure, respectively, on $R$; that is, those of the usual topology.

THEOREM 1.16. $\underline{A}=\left(A \cup A^{+}\right)$where $A^{+}$denotes the set of points $p \in R$ such that $p \notin A$, but there exists $\varepsilon_{\mathbb{1}}>0$ such that both

$$
(p-\varepsilon, p) \subseteq(\overline{p-\varepsilon, p) \cap A} \text {, and }(p, p+\varepsilon) \subseteq \overline{(p, p+\varepsilon) \cap A}
$$

for $0<\varepsilon<\varepsilon_{1}$. 
Proof. If $p \in \underline{A}$, then $p \in A$ or $p \notin A$. If $p \in A$, then $p \in\left(A \cup A^{+}\right)$. Thus, to demonstrate that $\underline{A} \subseteq\left(A \cup A^{+}\right)$, it remains only to show that if $p \in(\underline{A}-A)$ then $p \in A^{+}$. The proof of this is by contradiction. Assume that $p \notin A^{+}$. But by hypothesis $p \notin A$ so that for every $\varepsilon>0$,

$$
(p-\varepsilon, p) \nsubseteq \overline{(p-\varepsilon, p) \cap A} \quad \text { or } \quad(p, p+\varepsilon) \nsubseteq \overline{(p, p+\varepsilon) \cap A} .
$$

Consider the sequence $\left\{n^{-1}\right\}_{n=1}^{\infty}$. Then for any $n$,

$$
\left(p-n^{-1}, p\right) \nsubseteq \overline{\left(p-n^{-1}, p\right) \cap A} \quad \text { or } \quad\left(p, p+n^{-1}\right) \nsubseteq \overline{\left(p, p+n^{-1}\right) \cap A} .
$$

Now for each $n$, define the set $U_{n}$ as follows: If

let

$$
\left(p-n^{-1}, p\right) \nsubseteq \overline{\left(p-n^{-1}, p\right) \cap \bar{A}},
$$

$$
U_{n}=\left[\left(p-n^{-1}, p\right)-\overline{\left.\left(p-n^{-1}, p\right) \cap A\right]}\right.
$$

which is nonvoid and open. On the other hand, if for some values of $n$,

$$
\left(p-n^{-1}, p\right) \subseteq \overline{\left(p-n^{-1}, p\right) \cap A},
$$

then for these values of $n$,

and we let

$$
\left(p, p+n^{-1}\right) \nsubseteq \overline{\left(p, p+n^{-1}\right) \cap A}
$$

$$
U_{n}=\left[\left(p, p+n^{-1}\right)-\overline{\left(p, p+n^{-1}\right) \cap A}\right]
$$

which is nonvoid and open. Then letting $U=\bigcup_{n=1}^{\infty} U_{n}, U$ is an open set, and clearly $p \in \bar{U}$. Thus, $(U \cup\{p\})$ is semi-open, and note that $[(U \cup\{p\}) \cap A]=\varnothing$. Thus $A \subseteq[R-(U \cup\{p\})]$ which is semi-closed. This implies that $\underline{A} \subseteq[R-(U \cup\{p\})]$. Hence, $p \notin \underline{A}$, which is a contradiction. Thus $\underline{A} \subseteq\left(A \cup A^{+}\right)$.

If $p \in A$, then $p \in \underline{A}$. Thus to show that $\left(A \cup A^{+}\right) \subseteq \underline{A}$, it is only necessary to show that $A^{+} \subseteq \underline{C}$. This proof is accomplished by proving the contrapositive statement. If $p \notin \underline{A}$, it will be shown that $p \notin A^{+}$. If $p \notin \underline{A}$, then there is a semi-open set $S$ such that $p \in S$ and $(S \cap A)=\emptyset$. Since $S$ is semi-open, there exists an open subset, $O$, of $R$ such that $O \subseteq S \subseteq \bar{O}$. Thus $(O \cap A)=\emptyset$. There are two cases, either $p \in O$, or $p \in(S-0)$.

Case I. If $p \in O$, then there exists $\varepsilon>0$ such that $((p, p+\varepsilon) \cap A)=\emptyset$ and $((p-\varepsilon, p) \cap A)=\varnothing$ so that $p \notin A^{+}$.

Case II. If $p \in(S-O)$, then since $S \subseteq \bar{O}$, for every $\varepsilon>0$, either $((p-\varepsilon, p) \cap O) \neq \varnothing$ or $((p, p+\varepsilon) \cap O) \neq \emptyset$. If $((p-\varepsilon, p) \cap O) \neq \varnothing$, then
due to the fact that

$$
\overline{((p-\varepsilon, p) \cap A} \cap[O \cap(p-\varepsilon, p)])=\emptyset
$$

we have the fact that

$$
(p-\varepsilon, p) \nsubseteq \overline{(p-\varepsilon, p) \cap A} .
$$

On the other hand, if $((p-\varepsilon, p) \cap 0)=\emptyset$, then $((p, p+\varepsilon) \cap 0) \neq \emptyset$, and a similar argument shows that

$$
(p, p+\varepsilon) \nsubseteq \overline{(p, p+\varepsilon) \cap A} .
$$

Thus if $p \in(S-O), p \notin A^{+}$.

Therefore it has been shown that $\underline{A}=\left(A \cup A^{+}\right)$.

THEOREM 1.17. If $A \subset R$, and ( ) is the usual semi-closure operator on $R$, then $D_{A}$ is the set of points $p \in \bar{R}$ such that $p \& A$, but for any $\varepsilon>0$, either

$$
(\overline{(p-\varepsilon, p) \cap A})^{0} \neq \varnothing \quad \text { or } \quad(\overline{(p, p+\varepsilon) \cap A})^{0} \neq \varnothing,
$$

where $D_{A}$ is as in [2].

Proof. If we let $G_{A}=\{p \in R: p \notin A$, but for any $\varepsilon>0$, either $(\overline{(p-\varepsilon, p) \cap A})^{0} \neq \varnothing$ or $\left.(\overline{(p, p+\varepsilon) \cap A})^{0} \neq \varnothing\right\}$, it must be shown that $D_{A}=G_{A}$.

First, it will be shown that $D_{A} \subseteq G_{A}$. For this purpose, it suffices to show that if $p \notin G_{A}$, then $p \notin D_{A}$. If $p \notin G_{A}$ and $p \in A$, then $p \notin D_{A}$ since it was shown in [2] that $\left(A \cap D_{A}\right)=\emptyset$. If $p \notin G_{A}$ and $p \notin A$, then there exists an $\varepsilon_{1}>0$ such that

$$
\left(\overline{\left(p-\varepsilon_{1}, p\right) \cap A}\right)^{0}=\varnothing \quad \text { and } \quad\left(\overline{\left(p, p+\varepsilon_{1}\right) \cap A}\right)^{0}=\varnothing .
$$

Now let $F_{A}=\left(R-\left(p-\varepsilon_{1}, p+\varepsilon_{1}\right)\right)$, and consider $\underline{\left(A \cup F_{A} \cup B\right)}$ for arbitrary $B \subset R$.

If $q \in\left(\underline{\left.A \cup F_{A} \cup B\right)}\right.$, then either $q \in\left(A \cup F_{A} \cup B\right)$ or $q \notin\left(A \cup F_{A} \cup B\right)$. If $q \in\left(\left(A \cup F_{A} \cup B\right)-\left(A \cup F_{A} \cup B\right)\right)$, then $q \notin\left(A \cup F_{A} \cup B\right)^{+}$so that there is a number $e>0$ such that for $0<\varepsilon<e$,

and

$$
\overline{(q-\varepsilon, q) \cap\left(A \cup F_{A} \cup B\right)} \supseteq(q-\varepsilon, q)
$$

$$
\overline{(q, q+\varepsilon) \cap\left(A \cup F_{A} \cup B\right)} \supseteq(q, q+\varepsilon) .
$$

Also, since $q \notin\left(A \cup F_{A}\right)$, there exists $\dot{\varepsilon}_{2}=\min \left[q-\left(p-\varepsilon_{1}\right), p+\varepsilon_{1}-q\right]$ which is positive because $\left(p-\varepsilon_{1}\right)<q<\left(p+\varepsilon_{1}\right)$, such that for $0<\varepsilon \leqslant \varepsilon_{2}$,

$$
\left(\overline{(q-\varepsilon, q) \cap\left(A \cup F_{A}\right)}\right)^{0}=\emptyset \quad \text { and } \quad\left(\overline{(q, q+\varepsilon) \cap\left(A \cup F_{A}\right)}\right)^{0}=\emptyset .
$$

Now let $\varepsilon_{3}=\min \left[e, \varepsilon_{2}\right]$, and consider $0<\varepsilon<\varepsilon_{3}$. Then

$$
\begin{aligned}
(q-\varepsilon, q) \subset \overline{(q-\varepsilon, q) \cap\left(A \cup F_{A} \cup B\right)} & \\
& =\overline{\left[(q-\varepsilon, q) \cap\left(A \cup F_{A}\right)\right.} \cup \overline{(q-\varepsilon, q) \cap B]},
\end{aligned}
$$

Fundamenta Mathematicae, T. LXXIV 
so that

$$
[(q-\varepsilon, q)-\overline{(q-\varepsilon, q) \cap B}] \subseteq \overline{(q-\varepsilon, q) \cap\left(A \cup F_{A}\right)} .
$$

But

is open, and

$$
[(q-\varepsilon, q)-\overline{(q-\varepsilon, q) \cap B]}
$$

$$
\left(\overline{(q-\varepsilon, q) \cap\left(A \cup F_{A}\right)}\right)^{0}=\varnothing
$$

so that

$$
[(q-\varepsilon, q)-\overline{(q-\varepsilon, q) \cap B}]=\emptyset
$$

that is,

$$
(q-\varepsilon, q) \subseteq \overline{(q-\varepsilon, q) \cap B} \text { for } 0<\varepsilon<\varepsilon_{3} .
$$

In a similar fashion, $(q, q+\varepsilon) \subseteq \overline{(q, q+\varepsilon) \cap B}$ for $0<\varepsilon<\varepsilon_{3}$. Hence, since $q \notin B$, it follows from Theorem 1.16 that $q \in \underline{B}$. Thus,

$$
\underline{\left(A \cup F_{A} \cup B\right)} \subseteq\left(A \cup F_{A} \cup \underline{B}\right) \subseteq \underline{\left(A \cup F_{A} \cup \underline{B}\right) \subseteq \underline{\left(A \cup F_{A} \cup B\right)}}
$$

so that

$$
\underline{\left(A \cup F_{A} \cup B\right)}=\left(A \cup F_{A} \cup \underline{B}\right) \quad \text { for arbitrary } B \subseteq R .
$$

Hence $D_{A} \subseteq F_{A} \subseteq\left(R-\left(p-\varepsilon_{1}, p+\varepsilon_{1}\right)\right)$, so that $p \notin D_{A}$. Therefore, it has been demonstrated that $D_{A} \subseteq G_{A}$.

Secondly, it must be shown that if $p \in G_{A}$, then $p \in D_{A}$. If $p \in A^{+}$, then it follows from [2], that $A^{+} \subseteq D_{A}$ so that $p \in D_{A}$. Thus we will consider $p \in\left(G_{A}-A^{+}\right)$.

If $p \in G_{A}$, then $p \notin A$ and for any $\varepsilon>0$ either

$$
(\overline{(p-\varepsilon, p) \cap A})^{0} \neq \varnothing \quad \text { or } \quad(\overline{(p, p+\varepsilon) \cap A})^{0} \neq \varnothing .
$$

Clearly, if $\left(\overline{\left(p-\varepsilon_{1}, p\right) \cap A}\right)^{0}=\varnothing$, then $(\overline{(p-\varepsilon, p) \cap A})^{0}=\varnothing$ for $0<\varepsilon<\varepsilon_{1}$. Thus, either $(\overline{(p-\varepsilon, p) \cap A})^{0} \neq \emptyset$ for all $\varepsilon>0$, or $(\overline{(p, p+\varepsilon) \cap A})^{0} \neq \emptyset$ for all $\varepsilon>0$.

In addition, if $p \notin A^{+}$and $p \notin A$, then for all $\varepsilon>0$, either

$$
(p-\varepsilon, p) \nsubseteq \overline{(p-\varepsilon, p) \cap A} \quad \text { or } \quad(p, p+\varepsilon) \nsubseteq \overline{(p, p+\varepsilon) \cap A} .
$$

If $\left(p-\varepsilon_{1}, p\right) \subseteq \overline{\left(p-\varepsilon_{1}, p\right) \cap A}$, then

Thus, either

$$
(p-\varepsilon, p) \subseteq \overline{(p-\varepsilon, p) \cap A} \text { for } 0<\varepsilon \leqslant \varepsilon_{1} .
$$

$$
(p-\varepsilon, p) \nsubseteq \overline{(p-\varepsilon, p) \cap A} \quad \text { for all } \varepsilon>0
$$

or there exists $\varepsilon_{1}>0$ such that

$$
(p-\varepsilon, p) \subseteq \overline{(p-\varepsilon, p) \cap A} \text { for } 0<\varepsilon \leqslant \varepsilon_{1} .
$$

Now if $p \in\left(G_{A}-A^{+}\right)$, note that $p \notin A$ and $p \in \bar{A}$. Thus consider $B=(R-\bar{A})$; observe that $(A \cup B)=(R-(\bar{A}-A)) ;$ and by Theorem 0.6 ,

$$
\underline{(A \cup B)}=(\underline{R-(\bar{A}-A)})=R .
$$

Hence $p \in(A \cup B)$. Note that $p \in \bar{A}$, so that $p \notin B$.

Now it will be shown that $p \notin \underline{B}$. Without loss of generality, it will be assumed that

$$
(\overline{(p-\varepsilon, p) \cap A})^{0} \neq \varnothing \quad \text { for all } \varepsilon>0 .
$$

Then there are two cases: either

$$
(p-\varepsilon, p) \nsubseteq \overline{(p-\varepsilon, p) \cap A} \text { for all } \varepsilon>0,
$$

or there exists $\varepsilon_{1}>0$ such that

$$
(p-\varepsilon, p) \subseteq \overline{(p-\varepsilon, p) \cap A} \text { for } 0<\varepsilon \leqslant \varepsilon_{1} \text {. }
$$

Case I. For all $\varepsilon>0$,

$$
(\overline{(p-\varepsilon, p) \cap A})^{0} \neq \varnothing \quad \text { and } \quad(p-\varepsilon, p) \nsubseteq \overline{(p-\varepsilon, p) \cap A},
$$

and we wish to consider

$$
\begin{aligned}
((p-\varepsilon, p) \cap B) & =[(p-\varepsilon, p) \cap(R-\bar{A})] \\
& =((p-\varepsilon, p)-\bar{A}) \subseteq[(p-\varepsilon, p)-\overline{(p-\varepsilon, p) \cap \boldsymbol{A}}]
\end{aligned}
$$

since $\overline{(p-\varepsilon, p) \cap A} \subseteq \bar{A}$. Thus

$$
\begin{aligned}
\overline{(p-\varepsilon, p) \cap B} & \subseteq \overline{(p-\varepsilon, p)-\overline{(p-\varepsilon, p) \cap A}} \\
& \subseteq\left(\{p-\varepsilon\} \cup\{p\} \cup\left[(p-\varepsilon, p)-(\overline{(p-\varepsilon, p) \cap A})^{0}\right]\right) .
\end{aligned}
$$

Therefore, since

$$
(\overline{(p-\varepsilon, p) \cap A})^{0} \neq \varnothing, \quad(p-\varepsilon, p) \nsubseteq \overline{(p-\varepsilon, p) \cap B}
$$

for all $\varepsilon>0$; that is, $p \notin B^{+}$. Thus, in this case $p \notin \underline{B}$.

Case II. For all $\varepsilon>0,(\overline{(p-\varepsilon, p) \cap A})^{0} \neq \varnothing$, and there exists $\varepsilon_{1}>0$ such that

$$
(p-\varepsilon, p) \subseteq \overline{(p-\varepsilon, p) \cap A} \text { for } 0<\varepsilon \leqslant \varepsilon_{1} \text {. }
$$

Again, consider

$$
((p-\varepsilon, p) \cap B) \subseteq((p-\varepsilon, p)-\overline{(p-\varepsilon, p) \cap A})=\emptyset
$$


for $0<\varepsilon \leqslant \varepsilon_{1}$; that is, $p \notin B^{+}$. Thus in this case $p \notin \underline{B}$. Hence, if $p \in\left(G_{A}-A^{+}\right)$, defining $B=(R-\bar{A})$ we have $p \in \underline{(\underline{A \cup B})}$ while $p \notin A$ and $p \notin \underline{B}$. Thus since

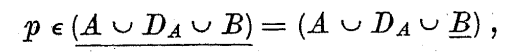

it follows that $p \in D_{A}$.

Consequently, it has been shown that $D_{A}=G_{A}$.

EXAMPLE 1.6. It follows then, from the last two theorems, that in the finest topology on the real numbers with the usual semi-open sets, the closed sets are those which contain every point having the property that arbitrary neighborhoods of the point in the usual topology intersect the set in sets whose closures have nonvoid interiors in the usual topology.

Thus, in this topology, the open sets are those which have the property that every point in the set has a sufficiently small neighborhood in the usual topology which intersects the complement of the set in a set which is nowhere dense in the usual topology. Therefore the finest topology on the real numbers for which the semi-open sets are those of the usual topology is the collection $\{O-N\}$ where $O$ is open in the usual topology and $N$ is nowhere dense in the usual topology. Notice that both $O$ and $N$ vary over all such choices.

Remark 1.7. The finest topology on the real numbers with the usual semi-open sets as described in Example 1.6 is not locally connected nor is it first countable, while the usual topology is locally connected and first countable. Thus local connectedness and first countability are not semi-topological properties.

2. Semi-topological classes. If $X$ is a set of points, let $T(X)$ denote the collection of all topological spaces which have $\dot{X}$ as their set of points.

DEFINITION 2.1. If $X$ is a set of points and if $(X, \tau)$ and $\left(X, \tau^{*}\right)$ are two elements of $T(X)$, then $(X, \tau)$ is semi-correspondent to $\left(X, \tau^{*}\right)$ if and only if $\mathrm{SO}(X, \tau)=\mathrm{SO}\left(X, \tau^{*}\right)$.

THEOREM 2.1. Semi-correspondent is an equivalence relation on the collection $T(X)$.

Proof. (1) Clearly $(X, \tau)$ is semi-correspondent to itself for any topology $\tau$ on $X$.

(2) Symmetry follows from the symmetry of set equality:

(3) Transitivity follows from the transitivity of set equality.

Thus the collection $T(X)$ of topological spaces is partitioned into equivalence classes. Let $[X, \mathrm{SO}(X, \tau)]$ denote the equivalence class of topological spaces with the same collection of semi-open sets as $(X, \tau)$. As was shown in $[2],[X, \mathrm{SO}(X, \tau)]$ contains a maximal topological space in the sense that the topology induced on $X$ by the semi-closure operator is finer than the topology on any other space in $[X, \mathrm{SO}(X, \tau)]$, and of course the topology so induced gives a topological space in $[X, \mathrm{SO}(X, \tau)]$.

Definition 2.2. If $(X, \tau)$ is a topological space, the finest topology on a space in $[X, \mathrm{SO}(X, \tau)]$ is denoted by $F(\tau)$.

DefinItIoN 2.2. The equivalence elasses of $T(X)$ under the relation of semi-correspondence will be called the semi-topological classes of $X$.

Remark 2.1. Although a semi-topological class contains a topological space with the finest topology, it need not contain a topological space with a coarsest topology, as is indicated by the following example.

EXAMPLE 2.1. Let $X=(0,1)$ and define $\tau_{1}$ and $\tau_{2}$ as follows. Let $\tau_{1}$ consist of $\varnothing$ and all sets of the form $\left(0,2^{-n}\right)$ for $n=0,1,2, \ldots$ Let $\tau_{2}$ consist of $\varnothing$ and all sets of the form $\left(0,3^{-n}\right)$ for $n=1,2,3, \ldots$

Clearly, both $\tau_{1}$ and $\tau_{2}$ are topologies on $X$. Note that in either case, the closure of any nonvoid open set is $X$. Furthermore, in either topology, a nonvoid set is semi-open if and only if it contains a set of the form: $(0, a)$ where $0<a \leqslant 1$. Thus $\left(X, \tau_{1}\right)$ is semi-correspondent to $\left(X, \tau_{2}\right)$, and both belong to the same semi-topological class. Note, however, that the only topology coarser than both $\tau_{1}$ and $\tau_{2}$ is the trivial topology. But $X$ together with the trivial topology does not belong to $\left[X, \operatorname{SO}\left(X, \tau_{1}\right)\right]$. Thus, semitopological classes do not necessarily contain a topological space minimal in the sense that its topology is the coarsest giving the same collection of semi-open sets.

The proofs of the following two theorems are immediate from the definitions.

THEOREM 2.2. If $f:(X, \tau) \rightarrow\left(Y, \tau^{*}\right)$ is irresolute, and if $\left(X, \tau_{1}\right)$ $\epsilon[X, \mathrm{SO}(X, \tau)]$ and $\left(Y, \tau_{1}^{*}\right) \epsilon\left[Y ; \operatorname{SO}\left(Y, \tau^{*}\right)\right]$, then $f:\left(Y, \tau_{1}\right) \rightarrow\left(Y, \tau_{1}^{*}\right)$ is irresolute.

THEOREM 2.3. If $f:(X, \tau) \rightarrow\left(Y, \tau^{*}\right)$ is semi-continuous, and if $\left(X, \tau_{1}\right)$ $\epsilon[X, \operatorname{SO}(X, \tau)]$, then $f:\left(X, \tau_{1}\right) \rightarrow\left(Y, \tau^{*}\right)$ is semi-continuous.

THEOREM 2.4. If $\left(X, \tau^{*}\right)$ is a $T_{2}$ space and $(X, \tau) \in\left[X, \operatorname{SO}\left(X, \tau^{*}\right)\right]$, then $(X, \tau)$ is $T_{2}$.

Proof. The proof of this theorem is given in a series of steps, thirteen of which, for lack of a better name, are called lemmas.

Without loss of generality, we may take the $T_{2}$ topology $\tau^{*}$ to be $F^{\prime}\left(\tau^{*}\right)$; see the discussion following Theorem 2.1. We may do this because if any topological space in $\left[X, \mathrm{SO}\left(X, \tau^{*}\right)\right]$ is $T_{2}$, the finest topology $F\left(\tau^{*}\right)$ is also $T_{2}$.

The proof is by contradiction. Assume that there is a space $(X, \tau)$ $\epsilon\left[X, \mathrm{SO}\left(X, \tau^{*}\right)\right]$ such that $\tau$ is not $T_{2}$. There are three cases:

(I) $\tau$ is $T_{1}$ but not $T_{2}$, 
(II) $\tau$ is $T_{0}$ but not $T_{1}$, or

(III) $\tau$ is not $T_{0}$.

Case I. If $\tau$ is $T_{1}$ but not $T_{2}$, then there exist distinct points $a$ and $b$ such that for all $A$ and $B$ in $\tau$ such that $a \in A$ and $b \in B$, we have $(A \cap B)$ $\neq \varnothing$. Since $\tau$ is $T_{1}$, we may choose $A$ and $B$ such that $b \notin A$ and $a \notin B$.

LEMNAa 2.1. Under these hypotheses, both $a$ and $b$ are in $(\overline{A \cap B})$, where $\overline{()}$ denotes the closure in $\tau$.

Proof of Lemma 2.1. Otherwise, say if

$$
a \notin \overline{(A \cap B)}, \quad(A \cap(X-\overline{A \cap B})) \epsilon \tau
$$

and is disjoint from $B$ while containing $a$. Similarly, if $b \notin(\overline{A \cap B})$, $(B \cap(X-\overline{A \cap B)}) \in \tau$ and is disjoint from $A$ while containing $b$.

Leñua 2.2. Under these hypotheses, $((A \cap B) \cup\{a, b\}) \in \mathrm{SO}\left(X, \tau^{*}\right)$.

Proof of Lemma 2.2. By Lemma 2.1, $(A \cap B) \subseteq((A \cap B) \cup\{a, b\})$ $\subseteq \overline{(A \cap B)}$, so that $((A \cap B) \cup\{a, b\}) \in \mathrm{SO}(X, \tau)=\mathrm{SO}\left(X, \tau^{*}\right)$.

Lewara 2.3. Under these hypotheses, both $a$ and $b$ are in $\overline{(A \cap B)^{*}}$, where ()$^{*}$ denotes the closure in $\tau^{*}$.

Proof of Lemma 2.3. Otherwise, either $a \notin \overline{(A \cap B)^{*}}, b \notin \overline{(A \cap B)^{*}}$, or neither $a$ nor $b$ is in $\overline{(A \cap B)^{*} \text {. }}$

If $a \notin \overline{(A \cap B)^{*}}$ and $b \in \overline{(A \cap B)^{*}}$, then since $((A \cap B) \cup\{a, b\})$ is $\mathrm{n} \operatorname{SO}\left(X, \tau^{*}\right)$, either $((A \cap B) \cup\{a\})$ or $((A \cap B) \cup\{a, b\})$ is in $\tau^{*}$. But note that

$$
\left.([A \cap B) \cup\{a\}] \cap\left[X-\overline{(A \cap B)^{*}}\right]\right)=\{a\},
$$

and

$$
\left.([A \cap B) \cup\{a, b\}] \cap\left[X-\overline{(A \cap B)^{*}}\right]\right)=\{a\}
$$

so that in either case, $\{a\} \in \tau^{*}$. Thus $\{a\} \in \mathrm{SO}(X, \tau)$, and it follows by Theorem 0.3 that $\{a\} \in \tau$, but this is impossible by the way in which $a$ and $b$ were chosen.

Similarly, it is impossible that $b \notin \overline{(A \cap B)})^{*}$ and $a \in \overline{(A \cap B)^{*}}$.

If $a \notin \overline{(A \cap B)^{*}}$ and $b \notin \overline{(A \cap B)^{*}}$, then since $((A \cap B) \cup\{a, b\})$ is in $\mathrm{SO}\left(X, \tau^{*}\right)$, either $((A \cap B) \cup\{a\})$ or $((A \cap B) \cup\{b\})$ or $((A \cap B) \cup$ $\cup\{a, b\})$ is in $\tau^{*}$. In a manner similar to that of the above part of the proof, it is impossible that $((A \cap B) \cup\{a\}) \in \tau^{*}$ or $((A \cap B) \cup\{b\}) \epsilon \tau^{*}$. If $((A \cap B) \cup\{a, b\}) \in \tau^{*}$, then as before $\{a, b\} \in \tau^{*}$. Thus $\{a, b\} \in \operatorname{SO}(X, \tau)$, and by Theorem 0.3 and the above argument it follows that $\{a, b\} \in \tau$. But $\{a, b\} \in \tau$ implies that $\{a\} \in \tau$ and $\{b\} \in \tau$ so that this is also impossible.
Hence both $a$ and $b$ are in $\overline{(A \cap B)^{*}}$. Thus the proof of Lemma 2.3 is complete.

Now there exist sets $C$ and $D$ in $\tau^{*}$ such that $a \in C, b \in D$, and $(C \cap D)=\varnothing$.

LEMMA 2.4. Undier these hypotheses, $(C \cap(A \cap B)) \neq \emptyset$ and $(D \cap$ $\cap(A \cap B)) \neq \varnothing$.

Proof of Lemma 2.4. If $(C \cap(A \cap B))=\emptyset$, then $\overline{(A \cap B)^{*}}$ $\subseteq(X-C)$ so that $a \notin \overline{(A \cap B)}^{*}$ which contradicts Lemma 2.3. Similarly, $(D \cap(A \cap B)) \neq \varnothing$. This completes the proof of Lemma 2.4.

The sets $C$ and $D$ may or may not be in $\tau$, but they are in $\operatorname{SO}(X, \tau)$. Thus there exist open sets $E$ and $F$ in $\tau \subseteq \tau^{*}$ such that $E \subseteq C \subseteq \bar{E}$ and $F \subseteq D \subseteq \bar{F}$. Note that $(\bar{E} \cap(A \cap B)) \neq \bar{\emptyset}$ and $(\bar{F} \cap(A \cap B)) \neq \emptyset$ by Lemma 2.4, and since $C \subseteq \bar{E}$ and $D \subseteq \bar{F}$.

LEMma 2.5. Under these hypotheses, $a \in \bar{F}$ and $b \in \bar{E}$.

Proof of Lemma 2.5. If $a \notin \bar{F}$, then $(A \cap B) \subseteq \bar{F}$ for otherwise $(A-\bar{F}) \epsilon \tau$ and $((A-\bar{F}) \cap B) \neq \emptyset$. Then by the same argument as in the lemmas above, $[((A-\bar{F}) \cap B) \cap D] \neq \varnothing$; that is $((A-\bar{F}) \cap D) \neq \varnothing$ but $D \subseteq \bar{F}$. Thus $(A \cap B) \subseteq \bar{F}$. But $(A \cap B) \subseteq \bar{F}$ implies that $((A-\bar{F}) \cap B)$ $=\varnothing$ which contradicts the way $a$ and $b$ were chosen. Thus $a \in \bar{F}$. Similarly $b \in \bar{E}$. This completes the proof of Lemma 2.5.

Thus $(F \cup\{a\})$ and $(E \cup\{b\})$ are semi-open in $\tau$, and in $\tau^{*}$.

Lemina 2.6. Under these hypotheses, $\{a\} \in \tau^{*}$ and $\{b\} \in \tau^{*}$.

Proof of Lemma 2.6. ( $F \cup\{a\})$ is semi-open in $\tau^{*}$ while $\bar{F}^{*} \subseteq(X-C)$; thus $(F \cup\{a\}) \in \tau^{*}$. Thus $((F \cup\{a\}) \cap C) \in \tau^{*}$. Similarly $\{b\} \in \tau^{*}$. This completes the proof of Lemma 2.6.

By Theorem 0.3 , since $\{a\} \in \tau^{*}$ and $\{b\} \in \tau^{*}$, we have $\{a\} \in \tau$ and $\{b\} \in \tau$ as before. But this contradicts the way in which $a$ and $b$ were chosen. Thus Case I leads to a contradiction.

Case II. If $\tau$ is $T_{0}$ but not $T_{1}$, there exists a pair of distinct points $a$ and $b$ such that for all $A$ and $B$ in $\tau$ such that $a \epsilon A$ and $b \in B$, we have $(A \cap B) \neq \varnothing$. Since $\tau$ is $T_{0}$ but not $T_{1}$, we may choose $A$ and $B$ such that $b \in A$ and $a \notin B$; that is, $a$ and $b$ are such that every neighborhood. of $a$ contains $b$, but there exists a neighborhood of $b$ which does not contain $a$.

LEMMA 2.7. Under these hypotheses, $a \in \overline{(A \cap B)}$.

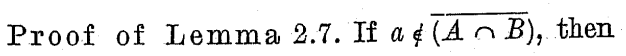

$$
(A \cap[X-\overline{(A \cap B)}]) \in \tau, \quad \text { and } \quad a \in((A \cap[X-\overline{(A \cap B)}])
$$


which is disjoint from $B$. This contradicts the hypothesis that every $\tau$-neighborhood of $a$ intersects $B$.

LEMMA 2.8. Under these hypotheses, $((A \cap B) \cup\{a\}) \in \mathrm{SO}\left(X, \tau^{*}\right)$.

Proof of Lemma 2.8. By Lemma 2.7, $(A \cap B) \subseteq((A \cap B) \cup\{a\})$ $\subseteq \overline{(A \cap B)}$ so that $((A \cap B) \cup\{a\}) \in \mathrm{SO}(X, \tau)=\mathrm{SO}\left(X, \tau^{*}\right)$.

LEMMA 2.9. Under these hypotheses, $a \in \overline{(A \cap B)^{*}}$.

Proof of Lemma 2.9. If $a \notin \overline{(A \cap B)^{*}}$, then $((A \cap B) \cup\{a\}) \epsilon \tau^{*}$. But then,

$$
\left.\left[((A \cap B) \cup\{a\}) \cap(X-\overline{(A \cap B)})^{*}\right)\right]=\{a\} \in \tau^{*} .
$$

As before, this leads to a contradiction. This completes the proof of Lemma 2.9 .

Now there exist sets $C$ and $D$ in $\tau^{*}$ such that $a \in O, b \in D$, and $(C \cap D)=\varnothing$. The sets $C$ and $D$ may or may not be in $\tau$, but they are in $\operatorname{SO}(X, \tau)$. Thus there exist open sets $E$ and $F$ in $\tau \subseteq \tau^{*}$ such that $E \subset C$ $\subseteq \bar{E}$ and $F \subseteq D \subseteq \bar{F}$.

Lemara 2.10. Under these hypotheses, $a \in \bar{F}$.

Proof of Lemma 2.10. If $a \notin \bar{F}$, then $(A \cap(X-\bar{F}))$ is an open set in $\tau$, containing a which does not contain $b$. This is a contradiction, and the proof of Lemma 2.10 is completed.

Thus $(F \cup\{a\})$ is semi-open in $\tau$ and in $\tau^{*}$.

Lema 2.11. Under these hypotheses, $\{a\} \in \tau^{*}$.

Proof of Lemma 2.11. ( $F \cup\{a\})$ is semi-open in $\tau^{*}$ while $\bar{F}^{*}$ $\subseteq(X-C)$. Thus, $(F \cup\{a\})$ is in $\tau^{*}$. Therefore, $((F \cup\{a\}) \cap C)=\{a\} \in \tau^{*}$. This completes the proof of Lemma 2.11.

By Theorem 0.3, since $\{a\} \in \tau^{*}$, we have $\{a\} \in \tau$ as before. This contradicts the way that $a$ and $b$ were chosen. Thus Case II leads to a contradiction.

Case III. If $\tau$ is not $T_{0}$, then there exist distinct points $a$ and $b$ such that every $\tau$-neighborhood of $a$ contains $b$, and every $\tau$-neighborhood of $b$ contains $a$; that is, for all $A$ and $B$ in $\tau$ such that $a \in A$ and $b \in B$, we have $(A \cap B) \supseteq\{a, b\}$.

Now there exist $C$ and $D$ in $\tau^{*}$ such that $a \in C, b \in D$, and $(C \cap D)=\varnothing$. The sets $C$ and $D$ may or may not be in $\tau$, but they are in $\operatorname{SO}(X, \tau)$. Thus there exist open sets $E$ and $F$ in $\tau \subseteq \tau^{*}$ such that $E \subseteq C \subseteq \bar{E}$ and $F \subseteq D \subseteq \bar{F}$. Note that $a \in \bar{E}$, and $b \in \bar{F}$.

Levara 2.12. Under these hypotheses, $a \in \bar{F}$ and $b \in \bar{E}$.

Proof of Lemma 2.12. If $a \notin \bar{F},(A \cap(X-\bar{F}))$ is an open set containing a which does not contain $b$, which is impossible. Similarly, $b \in \bar{E}$. This completes the proof of Lemma 2.12 .

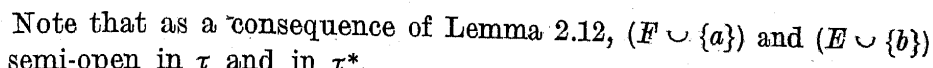
are semi-open in $\tau$ and in $\tau^{*}$.

LEMMA 2.13. Under these hypotheses, $\{a\} \in \tau^{*}$ and $\{b\} \in \tau^{*}$.

Proof of Lemma 2.13. ( $F \cup\{a\})$ is semi-open in $\tau^{*}$ while $\bar{F}^{*}$ $\subseteq(X-C)$. Thus $(F \cup\{a\}) \in \tau^{*}$. Therefore, $((F \cup\{a\}) \cap C)=\{a\} \in \tau^{*}$. Similarly, $\{b\} \in \tau^{*}$. This completes the proof of Lemma 2.13 .

By Theorem 0.3, since $\{a\}$ and $\{b\}$ are in $\tau^{*}$, we have $\{a\}$ and $\{b\}$ in $\tau$ as before. This contradicts the way in which $a$ and $b$ were chosen. Thus Case III leads to a contradiction.

Consequently, it has been shown that $\tau$ must be $T_{2}$.

THEOREM 2.5: If $f: X \rightarrow Y$ is a semi-homeomorphism, then $D_{f(A)}=f\left(D_{A}\right)$.

Proof. $D_{A}$ is the minimal set such that

$$
\underline{\left(A \cup D_{A} \cup B\right)}=\left(A \cup D_{A} \cup \underline{B}\right) \quad \text { for all } B \subseteq X .
$$

$D_{f(A)}$ is the minimal set such that

$$
\underline{\left(f(A) \cup D_{f(A)} \cup E\right)}=\left(f(A) \cup D_{f(A)} \cup \underline{E}\right) \quad \text { for all } E \subseteq Y .
$$

Note that in this proof, the same notation for semi-closure and the closure induced by the operator will be used in $X$ and $Y$.

First, it will be shown that

$$
\underline{\left(f(A) \cup f\left(D_{A}\right) \cup E\right)}=\left(f(A) \cup f\left(D_{A}\right) \cup \underline{E}\right) \quad \text { for "all } E \subseteq \underline{Y} \text {; }
$$

that is, $D_{f(A)} \subseteq f\left(D_{A}\right)$. Consider $\left(A \cup D_{A} \cup f^{-1}(E)\right)$ for arbitrary $E \subseteq Y$. Then

$$
\underline{\left(A \cup D_{A} \cup f^{-1}(E)\right)}=\left(A \cup D_{A} \cup \underline{f^{-1}(E)}\right)=\left(A \cup D_{A} \cup f^{-1}(\underline{E})\right)
$$

by Theorem 1.10. Also note that

$$
\begin{aligned}
\underline{f\left(A \cup D_{A} \cup f^{-1}(E)\right)} & =\underline{f\left(A \cup D_{A} \cup f^{-1}(E)\right)} \\
& =\underline{f(A) \cup f\left(D_{A}\right) \cup f\left(f^{-1}(E)\right)}=\underline{f(A) \cup f\left(D_{A}\right) \cup E}
\end{aligned}
$$

by Corollary 1.1 and the fact that $f$ is one-to-one and onto. But we also have,

$$
\begin{aligned}
f\left(A \cup D_{A} \cup f^{-1}(E)\right) & =f\left(A \cup D_{A} \cup f^{-1}(\underline{E})\right) \\
& =\left[f(A) \cup f\left(D_{A}\right) \cup f\left(f^{-1}(\underline{E})\right)\right]=\left[f(A) \cup f\left(D_{A}\right) \cup \underline{E}\right] .
\end{aligned}
$$

Hence, $D_{f(A)} \subseteq f\left(D_{A}\right)$. 
Next, it will be shown that $f^{-1}\left(D_{f(A)}\right) \supseteq D_{A}$; that is,

$$
\underline{\left(A \cup f^{-1}\left(D_{f(A)}\right) \cup B\right)}=\left(A \cup f^{-1}\left(D_{f(A)}\right) \cup \underline{B}\right) \quad \text { for all } B \subseteq X .
$$

Consider $\left(f(A) \cup D_{f(A)} \cup f(B)\right)$ for any $B \subseteq X$. Then

$$
\underline{f(A) \cup D_{f(A)} \cup f(B)}=\left(f(A) \cup D_{f(A)} \cup \underline{f(B)}\right)=\left(f(A) \cup D_{f(A)} \cup f(\underline{B})\right)
$$

by Corollary 1.1. Thus,

$$
\begin{aligned}
f^{-1}\left(\underline{\left.f(A) \cup D_{f(A)} \cup f(B)\right)}\right. & =f^{-i}\left(f(A) \cup D_{f(A)} \cup f(\underline{B})\right) \\
& =\left[f^{-1}(f(A)) \cup f^{-1}\left(D_{f(A)}\right) \cup f^{-1}(f(\underline{B}))\right] \\
& =\left[A \cup f^{-1}\left(D_{f(A)}\right) \cup \underline{B}\right] .
\end{aligned}
$$

But also,

$$
\begin{aligned}
f^{-1}\left(f(A) \cup D_{f(A)} \cup f(B)\right) & =\underline{f^{-1}\left(f(A) \cup D_{f(A)} \cup f(B)\right)} \\
& =\frac{f^{-1}(f(A)) \cup f^{-1}\left(D_{f(A)}\right) \cup f^{-1}(f(B))}{} \\
& =\underline{A \cup f^{-1}\left(D_{f(A)}\right) \cup B} .
\end{aligned}
$$

Thus $D_{A} \subseteq f^{-1}\left(D_{f(A)}\right)$. Hence, $f\left(D_{A}\right) \subseteq f\left(f^{-1}\left(D_{f(A)}\right)\right)=D_{f(A)}$, and together with the first part, this shows that $f\left(D_{A}\right)=D_{f(A)}$.

THEOREM 2.6. If $f:(X, \tau) \rightarrow(Y, \sigma)$ is a semi-homeomorphism, then $f:(X, F(\tau)) \rightarrow(Y, F(\sigma))$ is a homeomorphism, where $F(\tau)$. and $F(\sigma)$ are as defined in Definition 2.2.

Proof. $f$ is already known to be one-to-one and onto. If $A$ is any subset of $X$

$$
f\left(A^{k}\right)=f\left(A \cup D_{A}\right)=\left(f(A) \cup f\left(D_{A}\right)\right)=\left(f(A) \cup D_{f(A)}\right)=(f(A))^{k}
$$

by Theorem 2.5, where ()$^{k}$ denotes the closure induced as in [2], on both sets of points. Similarly, $f^{-1}\left(B^{k}\right)=\left(f^{-1}(B)\right)^{k}$ for any $B \subseteq Y$ since $f^{-1}$ is also a semi-homeomorphism. Thus $f$ is a homeomorphism, considered as a fuaction from $\left(X, F^{\prime}(\tau)\right)$ onto $(Y, F(\sigma))$.

THEOREM 2.7. $T_{2}$ is a semi-topological property.

Proof. If $f:(X, \tau) \rightarrow(Y, \sigma)$ is a semi-homeomorphism and $(X, \tau)$ is $T_{2}$, it must be shown that $(Y, \sigma)$ is $T_{2}$ also. By Theorem 2.4, $\left(X, F^{\prime}(\tau)\right)$ is $T_{2}$. Thus by Theorem 2.6, $(Y, F(\sigma))$ is $T_{2}$, and by Theorem 2.4,
$(Y, \sigma)$ is $T_{2}$.

THEOREM 2.8. If $A$ is dense in $(X, \tau)$, then $A$ is dense in $\left(X, F^{\prime}(\tau)\right)$.
Proof. If $A$ is dense in $(X, \tau)$, then $\bar{A}=X$. Furthermore, $A^{k} \subseteq \bar{A}=X$ where ()$^{k}$ denotes the closure in $\left(X, F^{\prime}(\tau)\right)$ as in [2]. It must be shown that $A^{k}=X$. The proof is by contradiction. Assume $A^{k} \subset X$, then $\left(X-A^{k}\right) \neq \varnothing$, and $\left(X-A^{k}\right) \in F^{\prime}(\tau)$. Thus $\left(X-A^{k}\right) \in \operatorname{SO}(X, \tau)$ so that by Theorem 0.3 , there exists a nonvoid open set $O \in \tau$ such that $O \subseteq\left(X-A^{k}\right)$. Also $(O \cap A)=\emptyset$, so that $A \subseteq(X-O) \subset X$. But this implies that $\bar{A}$ $\subseteq(X-0) \subset X$ which is a contradiction. Thus, $A^{k}=X$, and $A$ is dense in $(X, F(\tau))$.

THEOREM 2.9. Let $(X, \tau)$ and $(Y, \sigma)$ be topological spaces, let $f:(X, \tau)$ $\rightarrow(Y, \sigma)$ be a semi-homeomorphism, and let $A \subseteq X$ be dense in $(X, \tau)$. Then $f(A)$ is dense in $(Y, \sigma)$.

Proof. By theorem 2.8, $A$ is dense in $\left(X, F^{\prime}(\tau)\right)$. Furthermore, by Theorem 2.6, $f(A)$ is dense in $(Y, F(\sigma))$. We see from [2], that $(f(A))^{k} \subset \overline{f(A)}$. Thus, $f(A)$ is dense in $(Y, \sigma)$.

THEOREM 2.10. Separability is a semi-topological property.

Proof. If $X$ and $Y$ are topological spaces, $f: X \rightarrow Y$ is a semi-homeomorphism, and $X$ is separable, then there exists a countable dense subset $A$ of $X$. Then $f(A)$ is dense in $Y$ by Theorem 2.9 and countable since $f$ is one-to-one. Thus $Y$ is separable.

THEOREM 2.11. If $(X, \tau)$ is a topological space and $(X, F(\tau))$ is separated, then $(X, \tau)$ is also a separated space.

Proof. If $(X, F(\tau))$ is separated, then there exist sets $A$ and $B$ in $F(\tau)$ such that $A$ and $B$ are nonvoid, $(A \cup B)=X$, and $(A \cap B)=\emptyset$. Now $A$ and $B$ are in $\mathrm{SO}(X, \tau)$, so there exist nonvoid sets $O$ and $U$ in $\tau$ such that $O \subseteq A \subseteq \bar{O}$ and $U \subseteq B \subseteq \bar{U}$ where $\overline{()}$ denotes the closure in $(X, \tau)$. Then note that since $(A \cup B)=X,(\bar{O} \cup \bar{U})=X$.

Consider $(\bar{O} \cap \bar{U})$. If $(\bar{O} \cap \bar{U})=\emptyset$, then $(\bar{X}, \tau)$ is separated since $\bar{O}=(X-\bar{U})$ is open as well as closed.

On the other hand, if $x \in(\bar{O} \cap \bar{U})$, then it will be shown that $\{x\}$ is an open set in $\tau$. First, either $x \in A$ or $x \in B$. Without loss of generality, it will be assumed that $x \in A$, so that $x \notin B$. Now $U \subseteq B \subseteq \bar{U}$ and $x \in \bar{U}$ so that $U \subseteq(B \cup\{x\}) \subseteq \bar{U}$; that is, $(B \cup\{x\}) \in \operatorname{SO}(X, \bar{\tau})$. Thus $(B \cup\{x\})$ is semi-open in $(X, F(\tau))$. But $B$ is both open and closed in $(X, F(\tau))$, so that $\bar{B}=B$. Thus, since $(B \cup\{x\})$ is semi-open in $(X, F(\tau))$ it must be the case that $(B \cup\{x\}) \in F(\tau)$. Therefore, $[(B \cup\{x\}) \cap A] \epsilon F(\tau)$, but $[(B \cup\{x\}) \cap A]=\{x\}$. Thus $\{x\}$ is in $F(\tau)$. Hence $\{x\} \cdot \epsilon \mathrm{SO}(X, \tau)$. Thence by Theorem $0.3,\{x\} \in \tau$. Consequently, since $x$ was an arbitrary point of $(\bar{O} \cap \bar{U})$ it follows that every point of $(\bar{O} \cap \bar{U})$, considered as a singleton set, is open in $\tau$. Hence, since any union of open sets is open, $(\bar{O} \cap \bar{U})$ is open in $\tau$. But $\bar{O}$ and $\bar{U}$ are closed in $\tau$ so that $(\bar{O} \cap \bar{U})$ is also closed in $\tau$. 
Furthermore, $\bar{O} \subseteq(X-\bar{U})$ so that $(\bar{O} \cap \bar{U})$ is not $X$. Thus, $(\bar{O} \cap \bar{U})$ is both open and elosed, but it is neither empty nor the whole space. Hence, $(X, \tau)$ is separated.

THEOREM 2.12. Connected space is a semi-topological property.

Proof. If $(X, \tau)$ is connected, and $f:(X, \tau) \rightarrow(Y, \sigma)$ is a semi-homeomorphism, it must be shown that $(Y, \sigma)$ is connected. Now since $(X, \tau)$ is connected, it follows that $(X, F(\tau))$ is connected, for otherwise by Theorem 2.11, $(X, \tau)$ would be separated which would be a contradiction. Thus by Theorem 2.6, $(Y, F(\sigma))$ is connected and $F(\sigma)$ is finer than $\sigma$, $(\bar{X}, \sigma)$ is also connected.

Whereas it is not the case in general, that in a subspace of a topological space, a set will be semi-open if and only if it is the intersection of the subspace with a semi-open set in the larger space, we do have the following result.

THEOREM 2.13. If $(X, \tau)$ is a topological space and if $U$ is an open subset of $X$, then a set $S$ is semi-open in $(U, \tau \cap U)$, where $(\tau \cap U)$ denotes the relative topology on $U$, if and only if there exists a set $A \in \mathrm{SO}(X, \tau)$ such that $(A \cap U)=S$.

Proof. Necessity. If $S \subseteq U$ is semi-open in $(U, \tau \cap U)$, then there is a set $O \epsilon(\tau \cap \bar{U})$ such that $\bar{O} \subseteq S \subseteq \bar{O}^{r}$ where $\overline{()^{r}}$ denotes the closure in the relative topology. But $O$ is open in $(X, \tau)$ since. $U$ is open and $\bar{O}^{r} \subseteq \bar{O}$. Thus $O \subseteq S \subseteq \bar{O}$ and $S$ is semi-open in $(X, \tau)$.

Sufficiency. If $S=(A \cap U)$ where $A \in \operatorname{SO}(X, \tau)$, then there is a set $O \in \tau$ such that $O \subseteq A \subseteq \bar{O}$. Note that $(U \cap O) \subseteq(A \cap U)=S$. Now it remains to be shown that $S \subseteq \underline{(U \cap O)^{r}}$. If $N$ is any open set of $(U, \tau \cap U)$ containing an arbitrary point $p$ in $S$, not in $(U \cap O)$, then $N$ is open in $(X, \tau)$ and $S=(A \cap U) \subseteq \bar{O}$ so that $((N-\{p\}) \cap O) \neq \varnothing$. Then since $N \subseteq U,((N-\{p\}) \cap(\dot{O} \cap U)) \neq \emptyset$, so that $p \epsilon \overline{(O \cap u)^{r}}$. Thus $S \subseteq \overline{(\bar{U} \cap)^{r}}$, and $S$ is semi-open in $(U, \tau \cap U)$.

Whereas it has been demonstrated that the image of a connected set under a semi-homeomorphism is not necessarily connected, we do have the following result.

THEOREM 2.14. If $f:(X, \tau) \rightarrow(Y, \sigma)$ is a semi-homeomorphism, and if $U$ is an open, connected subset of $(X, \tau)$, then $f(U)$ is a conneced subset of $(\bar{X}, \sigma)$.

Proof. The following two lemmas will be useful in this proof.

LEMMA 2.14. If $O$ is an open connected subset of $(X, \tau)$, then $O$ is open and connected in $(X, F(\tau))$.

Proof of Lemma 2.14. Now $O$ is also open in $(X, F(\tau))$. Thus, by Theorem 2.13, the semi-open subsets in $(0, \tau \cap 0)$ and $(O, F(\tau) \cap O)$ are the same. Therefore; the identity function on $O$ is a semi-homeomorphism, and by Theorem 2.12, $O$ is connected in $\left(O, F^{\prime}(\tau) \cap 0\right)$. Thus $O$ is connected in $(X, F(\tau))$.

LEMVA 2.15. If $O$ is an open connected subset of $(Y, F(\sigma))$, then $O$ is connected in $(Y, \sigma)$.

Proof of Lemma 2.15. It will be shown that $\mathrm{SO}(O, F(\sigma) \cap O)$ $=\mathrm{SO}(O, \sigma \cap O)$ in which event the identity on $O$ is a semi-homeomorphism and $O$ is connected in $(O, \sigma \cap O)$, and thus $O$ is connected in $(Y, \sigma)$.

If $S \in \mathrm{SO}(O, F(\sigma) \cap O)$, then $S=(A \cap O)$ where $A \in \mathrm{SO}\left(Y, F^{\prime}(\sigma)\right)$ by Theorem 2.13. Thus since by Theorem $0.5 \quad(A \cap O) \in \operatorname{SO}(Y, F(\sigma))$ $=\mathrm{SO}(\underline{Y}, \sigma)$, we have an open set $V \epsilon \dot{\sigma}$ such that $V \subseteq(A \cap 0) \subseteq \bar{D}$ where () denotes the closure in $(Y, \sigma)$. Then $V$ is also open in $(O, \sigma \cap O)$. Furthermore, if $p \in((A \cap O)-V)$ and $N$ is any open set of $(O, \sigma \cap O)$ containing $p, N=(M \cap 0)$ where $M \in \sigma$ so that $((M-\{p\}) \cap \nabla) \neq \varnothing$ since $p \in \bar{V}$. Thus, since $V \subseteq O,((N-\{p\}) \cap V) \neq \varnothing$ so that $p \in \bar{V}^{r}$, where ${\overline{()^{r}}}^{r}$ denotes the closure in $(O, \sigma \cap O)$. Thus $V \subseteq(A \cap O) \subseteq \bar{V}^{r}$, and $(A \cap O)=S \in \mathrm{SO}(O, \sigma \cap O)$. Hence, SO $(O, F(\sigma) \cap O) C \mathrm{SO}(O, \sigma \cap O)$.

If $S \in \mathrm{SO}(O, \sigma \cap O)$, then there exists an open set $B \in(\sigma \cap O)$ such that $B \subseteq S \subseteq \bar{B}^{r}$. Now since $B \in(\sigma \cap O)$, there is an open set $W \epsilon \sigma$ such that $B=(\bar{O} \cap W)$. Also, $W \subseteq(W \cup S) \subseteq \bar{W}$ since $S \subseteq \bar{B}^{r} \subseteq \bar{B} \subseteq \bar{W}$. Thus

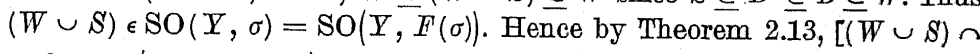
$\cap O] \in \mathrm{SO}(O, F(\sigma) \cap O)$. That is, $[(W \cup S) \cap O]=[(W \cap O) \cup(S \cap O)]$ $=(B \cup S)=S$ is in $\mathrm{SO}(O, F(\sigma) \cap O)$, and $\mathrm{SO}(O, \sigma \cap O) \subseteq \mathrm{SO}(O, F(\sigma) \cap O)$ so that equality holds, and the proof of Lemma 2.15 is complete.

Proof of Theorem 2.14. If $U$ is an open connected subset of $(X, \tau)$ and $f:(X, \tau) \rightarrow(Y, \sigma)$ is a semi-homeomorphism, then by Lemma 2.14, $U$ is open and connected in $(X, F(\tau))$. By Theorem 2.6, then, $f(U)$ is open and connected in $(Y, F(\sigma))$. Thus, by Lemma $2.15, f(U)$ is connected in $(Y, \sigma)$.

Remark 2.2. As a result of Theorem 2.13, Theorem 2.14, and the part of the proof of Lemma 2.15 which demonstrates that if $O \in F(\sigma)$, then $\mathrm{SO}(O, F(\sigma) \cap O)=\mathrm{SO}(O, \sigma \cap O)$ we have the following two corollaries:

CoROLLARY 2.1. If $(X, \tau)$ is a topological space and if $U \epsilon F^{\prime}(\tau)$ then $S$ is semi-open in $(U, \tau \cap U)$ if and only if there exists a set $A \in \operatorname{SO}(X, \tau)$ such that $(A \cap U)=S$.

COROLLARY 2.2. If $f:(X, \tau) \rightarrow(Y, \sigma)$ is a semi-homeomorphism, and if $U \in F(\tau)$ and $U$ is connected in $(X, \tau)$ then $f(U)$ is a connected subset of $(Y, \sigma)$. 


\section{References}

[1] Norman L. Levine, Semi-open sets and semi-continuity in topological spaces, Amer. Math. Monthly 70 (1963), pp. 36-41.

[2] S. Gene Crossley and S. K. Hildebrand, Semi-closure, Texas J. Sci. $22(2+3)$ (1971), pp. 99-112.

[3] - - Semi-closed sets and semi-continuity in topological spaces, Texas J. Sci. $22(2+3)$ (1971), pp. 123-126.

Reçu par la Rédaction le 4. 1. 1971
Errata to the paper

"Some properties of convex metric spaces"

Funđamenta Mathematicae 73 (1972), pp. 193-198

by

B. Krakus (Stockholm)

Page $194^{2-4}$. The words: "Since the set $L(X)$ is a boundary set (see [3]) and $L(X) \supset F(X)$ (see [4]), for every point $p \in S(X)$ there exist numbers ..."

should be replaced by the words: "It is known (see [8]) that $\operatorname{Int} S(X) \neq 0$ and that $L(X) \supset F(X)$ (see [4]). Hence there exist a point $p \in S(X)$ and numbers ...". 